\title{
Digital PET/CT allows for shorter acquisition protocols or reduced radiopharmaceutical dose in $\left[{ }^{18} \mathrm{~F}\right]-\mathrm{FDG}$ PET/CT
}

\author{
Ian Alberts ${ }^{1}$ (1) $\cdot$ Christos Sachpekidis $^{1} \cdot$ George Prenosil $^{1} \cdot$ Marco Viscione $^{1} \cdot$ Karl Peter Bohn $^{1} \cdot$ Clemens Mingels $^{1}$. \\ Kuangyu Shi ${ }^{1} \cdot$ Ali Ashar-Oromieh $^{1} \cdot$ Axel Rominger $^{1}$
}

Received: 14 October 2020 / Accepted: 18 January 2021 / Published online: 7 February 2021

(c) The Author(s) 2021

\begin{abstract}
Purpose To establish the feasibility of shorter acquisition times (and by analogy, applied activity) on tumour detection and lesion contrast in digital PET/CT.

Methods Twenty-one randomly selected patients who underwent oncological $\left[{ }^{18} \mathrm{~F}\right]$-FDG PET/CT on a digital PET/CT were retrospectively evaluated. Scan data were anonymously obtained and reconstructed in list-mode acquisition for a standard $2 \mathrm{~min} / \mathrm{bed}$ position (bp), $1 \mathrm{~min} / \mathrm{bp}$ and $30 \mathrm{~s} / \mathrm{bp} \mathrm{(100 \% ,50 \%} \mathrm{and} \mathrm{25 \%} \mathrm{time} \mathrm{or} \mathrm{applied} \mathrm{activity,} \mathrm{respectively).} \mathrm{Scans} \mathrm{were}$ randomized and read by two nuclear medicine physicians in a consensus read. Readers were blind to clinical details. Scans were evaluated for the number of pathological lesions detected. Measured uptake for lesions was evaluated by maximum and mean standardized uptake value (SUVmax and SUVmean, respectively) and tumour-to-backround ratio (TBR) were compared. Agreement between the three acquisitions was compared by Krippendorf's alpha.

Results Overall $n=100$ lesions were identified in the $2 \mathrm{~min}$ and $1 \mathrm{~min} / \mathrm{bp}$ acquisitions and $n=98$ lesions in the $30 \mathrm{~s} / \mathrm{bp}$ acquisitions. Agreement between the three acquisitions with respect to lesion number and tumour-to-background ratio showed almost perfect agreement (K's $\alpha=0.999)$. SUVmax, SUVmean and TBR likewise showed $>98 \%$ agreement, with longer acquisitions being associated with slightly higher mean TBR $(2 \mathrm{~min} / \mathrm{bp} 7.94 \pm 4.41$ versus $30 \mathrm{~s} / \mathrm{bp} 7.84 \pm 4.22, p<0.05)$.

Conclusion Shorter acquisition times have traditionally been associated with reduced lesion detectability or the requirement for larger amounts of radiotracer activity. These data confirm that this is not the case for new-generation digital PET scanners, where the known higher sensitivity results in clinically adequate images for shorter acquisitions. Only a small variation in the semi-quantitative parameters SUVmax, SUVmean and TBR was seen, confirming that either reduction of acquisition time or (by analogy) applied activity can be reduced as much as $75 \%$ in digital PET/CT without apparent clinical detriment.
\end{abstract}

Keywords List mode acquisition $\cdot$ PET/CT $\cdot$ Positron emission tomography $\cdot$ Digital PET

\section{Introduction}

The recent introduction of digital PET/CT scanners for routine clinical use represents a significant milestone for nuclear medicine and molecular imaging. Although PET/ CT scanners have always been truly "digital" insofar as their outputs were in the form of digital signals, the replacement of analogue photomultiplier tubes with solid-state detection systems resulted in the first fully "digital" PET/CT, and has

Ian Alberts

ian.alberts@insel.ch

1 Department of Nuclear Medicine. Inselspital, Bern University Hospital, University of Bern, Freiburgstr. 18, 3010 Bern, Switzerland surmounted many of the inherent physical limits placed by previous-generation analogue technologies. These new fully digital systems exhibit a plethora of technical advantages, which include a better coupling between the crystal and photodetectors, improved background-to-noise, faster time-offlight (TOF) and associated advanced TOF reconstruction. In addition, state-of-the-art digital systems often include longer axial coverage, smaller crystals, and more advanced electronics, which lead to higher sensitivity, higher spatial resolution, and shorter deadtime. These improved performance characteristics have been confirmed by a number of publications [1,2], which correspond to improvements in image quality and lesion detection [2-6].

Whereas the current iteration of the European Asociation of Nuclear Medicine (EANM) guidelines report examination 
protocols for analogue scanners and for "step-and-shoot" type acquisitions [7], no current guidelines are yet available for systems with digital acquisition. Although the performance characteristics, and EANM Research Ltd (EARL) compliance of such scanners have been reported [8-10], the full clinical potential of digital scanners is yet to be fully characterized. In particular, whereas the manufacturers' literature report shorter-acquisition times and/or reduced activity as potential improvements of such systems, the advantage for the patient needs to be investigated and verified in a clinical setting.

The aim of this present study is to assess the effect of short acquisition times or reduced activity on lesion detectability as assessed by two nuclear medicine physicians, followed by lesion uptake quantitation in a digital scanner.

\section{Materials and methods}

\section{Patient population}

In this retrospective analysis we included 21 randomly selected individuals who were examined on our digital PET/
CT (dPET/CT) at the University Hospital Bern, Inselspital and whose PET datasets were available for anonymous analysis. All patients underwent a clinically routine $\left[{ }^{18} \mathrm{~F}\right]-\mathrm{FDG}$ $\mathrm{PET} / \mathrm{CT}$ for oncological purposes. Patient characteristics are outlined in Table 1.

\section{Imaging protocol}

Patients were required to fast for $>6 \mathrm{~h}$ prior to scanning and finger-prick blood glucose measurement prior to scanning confirmed a venous blood glucose of $<120 \mathrm{mg} / \mathrm{dl}$. $3.5 \mathrm{MBq} /$ $\mathrm{kg}$ of $\left[{ }^{18} \mathrm{~F}\right]-\mathrm{FDG}$ was applied intravenously as per clinical routine. Scans were acquired at $60 \mathrm{~min}$ post injection of radiotracer (p.i.). All patients received regular whole-body PET scans (from skull base to the thighs) on a BiographVision 600 Edge PET/CT digital scanner (Siemens, Erlangen, Germany). A non-contrast-enhanced CT scan was performed $1 \mathrm{~h}$ post tracer injection with slice thickness of $1.0 \mathrm{~mm}$, pitch factor 1 , bone and soft tissue reconstruction kernels and maximum of $120 \mathrm{kV}$ and $90 \mathrm{mAs}$ by applying CARE $\mathrm{kV}$ and CARE Dose. Immediately after CT scanning, a whole-body PET (skull base to thighs) was acquired in 3D (matrix: $440 \times 440$ ) with a zoom factor of 1.0 . The
Table. 1 Number of lesions detected by dPET/CT at $2 \mathrm{~min} /$ bed position (bp), $1 \mathrm{~min} / \mathrm{bp}$ and $30 \mathrm{~s} / \mathrm{bp}$ list-mode acquisition and patient characteristics

\begin{tabular}{|c|c|c|c|c|c|c|c|c|}
\hline \multirow[t]{2}{*}{ Patient } & \multicolumn{3}{|c|}{ Number of pathological lesions } & & \multicolumn{3}{|c|}{ Patient characteristics } & \multirow[t]{2}{*}{ BMI } \\
\hline & $30 \mathrm{~s} / \mathrm{bp}$ & $1 \mathrm{~min} / \mathrm{bp}$ & $2 \mathrm{~min} / \mathrm{bp}$ & & Dose $(\mathrm{MBq})$ & $\mathrm{mSV}$ & Weight & \\
\hline 1 & 1 & 1 & 1 & & 217 & 4.1 & 63 & 21.0 \\
\hline 2 & 13 & 13 & 13 & & 234 & 4.4 & 64 & 21.6 \\
\hline 3 & 9 & 10 & 10 & & 234 & 4.4 & 69 & 20.3 \\
\hline 4 & 1 & 1 & 1 & & 304 & 5.7 & 86 & 25.9 \\
\hline 5 & 0 & 0 & 0 & & 132 & 2.5 & 38 & 15.6 \\
\hline 6 & 1 & 1 & 1 & & 385 & 7.3 & 100 & 27.7 \\
\hline 7 & 1 & 1 & 1 & & 166 & 3.1 & 46 & 17.5 \\
\hline 8 & 3 & 3 & 3 & & 333 & 6.3 & 99 & 28.9 \\
\hline 9 & 3 & 3 & 3 & & 175 & 3.3 & 48 & 17.6 \\
\hline 10 & 0 & 0 & 0 & & 215 & 4.0 & 60 & 20.5 \\
\hline 11 & 1 & 1 & 1 & & 338 & 6.4 & 98 & 31.6 \\
\hline 12 & 20 & 20 & 20 & & 321 & 6.0 & 92 & 35.0 \\
\hline 13 & 10 & 11 & 11 & & 259 & 4.9 & 73 & 26.8 \\
\hline 14 & 1 & 1 & 1 & & 230 & 4.3 & 62 & 23.3 \\
\hline 15 & 20 & 20 & 20 & & 342 & 6.4 & 100 & 30.8 \\
\hline 16 & 7 & 7 & 7 & & 219 & 4.1 & 55 & 21.4 \\
\hline 17 & 0 & 0 & 0 & & 266 & 5.0 & 75 & 23.1 \\
\hline 18 & 1 & 1 & 1 & & 312 & 5.9 & 89 & 25.7 \\
\hline 19 & 1 & 1 & 1 & & 308 & 5.8 & 86 & 28.7 \\
\hline 20 & 4 & 4 & 4 & & 167 & 3.1 & 46 & 18.9 \\
\hline 21 & 1 & 1 & 1 & & 256 & 4.8 & 75 & 24.2 \\
\hline Total & 98 & 100 & 100 & Mean & 257.7619 & 4.8 & 72.57143 & 24.1 \\
\hline
\end{tabular}

Dose applied dose in MBq, effective dose for an adults in $m S V$ effective dose for an adult $\left(1.9 \times 10^{-2} \mathrm{mSv} /\right.$ MBq [7]), weight (kg), BMI $\left(\mathrm{kg} / \mathrm{m}^{2}\right)$ 
emission data were corrected for randoms, scatter and decay. Reconstruction was conducted with TrueX (Point Spread Function, PSF) + time-of-flight (TOF) algorithm and $2 \mathrm{~mm}$ Gauss-filter was applied. TrueX is an iterative resolution recovery algorithm with PSF modelling included. Attenuation correction was performed using the low dose nonenhanced computed tomography data. Images were obtained in list mode. PET data were reprocessed to produce sinograms corresponding to $2 \mathrm{~min} / \mathrm{bed}$ position (bp), $1 \mathrm{~min} / \mathrm{bp}$, and $30 \mathrm{~s} / \mathrm{bp}$.

\section{Image evaluation}

Image analysis was performed using an appropriate workstation and software (SyngoVia; Siemens, Erlangen, Germany). Two experienced physicians (one board certified nuclear medicine physician and one experienced resident, both with experience in reading digital PET/CT scans) read all scans together. Disagreements were resolved by consensus. Readers were blinded to patient demographics and clinical details when reviewing scans. Neither reader had previously read or had familiarity with any of the included patients. The order of the scans was randomized prior to each read. Starting first with the reconstructions assumed to deliver the lowest quality, we analysed the $30 \mathrm{~s} / \mathrm{bp}$ acquisitions with respect to number of pathological lesions. After a $48 \mathrm{~h}$ waiting period, the scans were then re-randomized and the $1 \mathrm{~min} / \mathrm{bp}$, and likewise the $2 \mathrm{~min} / \mathrm{bp}$ scans analysed. The number of lesions judged to be pathological were counted, using previously published interpretation criteria [7]. To avoid discrepancies in the counting of polymetastatic individuals, a maximum of 20 lesions were counted per individual. Insofar as possible, recall bias was limited by reading each set of reconstructions starting first with the presumed lowest quality, with scans in randomized order, anonymized to patient demographics and with a $48 \mathrm{~h}$ waiting period between each reading session.

Scans were then re-analysed by both readers, this time comparing all three reconstructions with respect to lesion radiotracer uptake concomittantly. In patients with multiple lesions, up to five of the most visually prominent lesions in terms of radiotracer uptake were analysed to prevent overrepresentation by polymetastastic individuals. Lesion uptake was calculated by placing a volume-of-interest (VOI) around the lesion with $40 \%$ isocontour as previously published [11] and SUVmax and SUVmean were recorded. Background uptake was measured by placing a $14 \mathrm{~cm}^{3}$ volume-of-interest in normal liver tissue in the right liver lobe as previously described [12]. Tumour-to-background ratio (TBR) was defined as SUVmax (lesion)/UVmean (background) and taken as a measurement of lesion contrast and visibility. Regions of interest were copied and pasted between different list-mode acquisitions, ensuring that the same volumeof-interest was analysed for each acquisition.

\section{Statistical analysis}

Statistical analyses were performed using Excel (Microsoft, Redmond, Washington) and Graphpad Prism Version 6 (San Diego, California).

Agreement between the three acquisitions for number of lesions detected and for TBR were compared by Krippendorf's $\alpha$. Paired differences between acquisitions for lesion TBR, SUVmax and SUVmean were compaired by the paired student's $t$-test with correction for a log-normal distribution and Bonferonni correction, $p$ values $<0.05$ were considered statistically significant.

\section{Sample size estimate}

The null hypothesis is that the correlation in terms of lesion detection between the $2 \mathrm{~min} / \mathrm{bp}$ and $30 \mathrm{~s} / \mathrm{bp}$ scans is $<0.65$, the alternative hypothesis is that the correlation is $>0.65$. For a two sided $\alpha=0.05$ and $\beta=0.1$, a sample of $n=20$ was calculated. $N=21$ patients were included. Inclusion criteria were patients undergoing a routine oncological PET/CT in our department. Exclusion criteria were aborted scans, non-adherence to fasting requirements or initial blood glucose $>120 \mathrm{mg} / \mathrm{dl}$.

\section{Results}

\section{Lesion detection rate}

A total of $n=100$ malignant lesions were counted in $n=18$ individuals, with two polymetastatic individuals $(n>20$ lesions, with a maximum of 20 lesions analysed). Negative scans were observed for $n=3$ indviduals. Of the $n=100$ lesions, 100 were detected at $2 \mathrm{~min} / \mathrm{bp}$ and $1 \mathrm{~min} / \mathrm{bp}$ acquisition, and 98 at $30 \mathrm{~s} / \mathrm{bp}$; these two lesions missed in the $30 \mathrm{~s} /$ bp acquisitions were in polymetastatic individuals and would not have influenced the patient's staging or further management. In terms of lesion detection, the correlation between the $30 \mathrm{~s} / \mathrm{bp}$ and $2 \mathrm{~min} / \mathrm{bp}$ was therefore $98 \%$, confirming the alternative hypothesis. The results are shown in Table 1.

\section{Lesion detectability}

Lesion detectability was compared semi-quantiatively for the three acquisition modes by calculation of the tumour-to-background ratio (TBR) as described above for $n=49$ lesions. The results are shown in Fig. 1. Slightly higher mean TBR was seen at $2 \mathrm{~min} / \mathrm{bp}$ compared to $1 \mathrm{~min} / \mathrm{bp}(7.94 \mathrm{vs} 7.76, p=0.02)$ with no difference between $1 \mathrm{~min} / \mathrm{bp}$ and $30 \mathrm{~s}$ acquisitions (7.76 vs $7.84, p=0.29$ ). Overall, agreement between the three 
Fig. 1 Tumour-to-background ratio for the three acquisitions [30 s/bed position (bp); $1 \mathrm{~min} / \mathrm{bp} ; 2 \mathrm{~min} / \mathrm{bp}]$. The bars represent the standard error in the mean. No clear relationship between list-mode acquisition length and TBR was observed

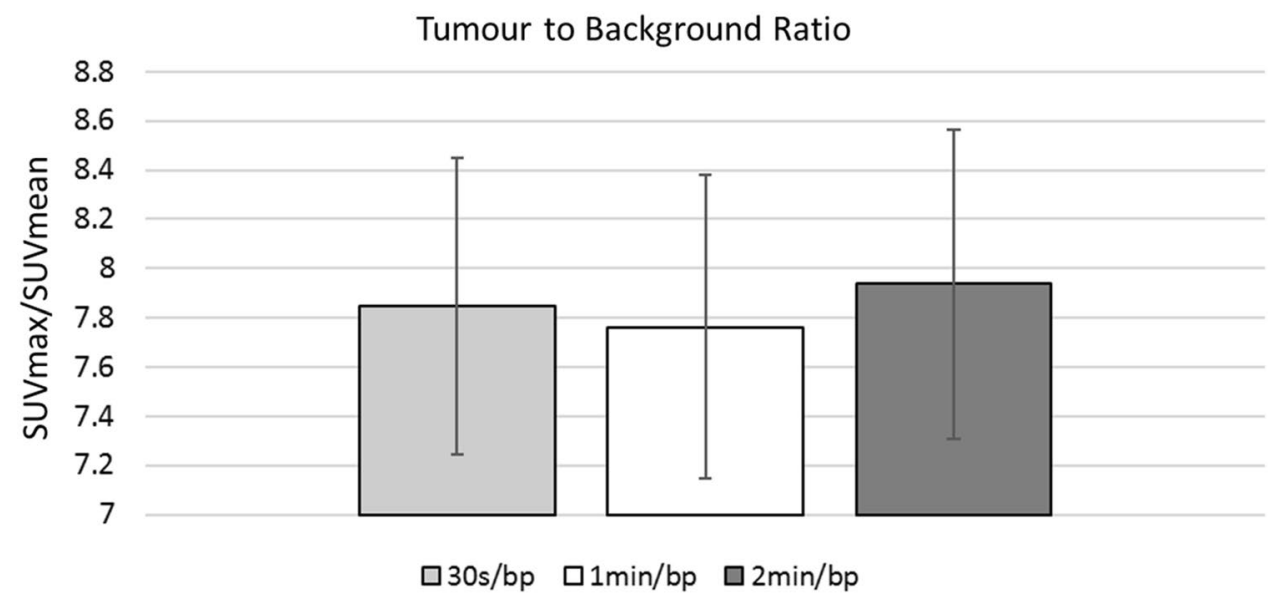

Table 2 Measured lesion SUVmax and lesion SUVmean ( \pm standard deviation, SD) for the three acquisitions

\begin{tabular}{llll}
\hline SUV max & Acquisition & Mean & SD \\
(Lesion) & $30 \mathrm{~s}$ & 15.11 & 9.38 \\
\hline SUV mean & $1 \mathrm{~min}$ & 14.62 & 9.30 \\
& $2 \mathrm{~min}$ & 14.99 & 8.73 \\
\hline (Lesion) & $30 \mathrm{~s}$ & 8.57 & 5.38 \\
\hline SUV mean & $1 \mathrm{~min}$ & 8.51 & 5.46 \\
\hline (Background) & $2 \mathrm{~min}$ & 8.73 & 5.60 \\
& $30 \mathrm{~s}$ & 2.05 & 0.27 \\
\hline
\end{tabular}

acquisitions was high $(\mathrm{K}$ 's alpha $=0.99)$. Mean variability between the $30 \mathrm{~s}$ and $2 \mathrm{~min} / \mathrm{bp}$ acquisitions was $0.79 \%$.

\section{Lesion uptake}

Lesion SUVmax and SUVmean were considered between the three acquistions, and is shown in Table 2. Slightly higher SUVmean was observed for the $2 \mathrm{~min} / \mathrm{bp}$ acquisitions compared to $30 \mathrm{~s} / \mathrm{bp}(p=0.0001)$. In Fig. 4 we show SUVmax at different time points and mean SUVmax. Paired student's $t$-test with Bonferonni correction reveal no signfificant differences between $30 \mathrm{~s}$ and $2 \mathrm{~min}, p>0.05$ for TBR. Likewise, no significant differences are seen for SUVmax (30 vs. $2 \mathrm{~min} ; p>0.05$ ). Mean variability between the $30 \mathrm{~s}$ and $2 \mathrm{~min} / \mathrm{bp}$ acquisitions was $0.35 \%$ for SUVmean and $0.77 \%$ for SUVmax.

\section{Discussion}

In $n=21$ patients undergoing routine oncological $\left[{ }^{18} \mathrm{~F}\right]-\mathrm{FDG}$ PET/CT on a digital scanner, we find that $98 \%$ of lesions $(n=98 / 100)$ are detected at a table velocity equivalent to $30 \mathrm{~s} / \mathrm{bp}$ ( $25 \%$ of standard acquisition time). For the two "missed" lesions, we note that these were in polymetastatic individuals where the extra lesions could be slightly better discriminated, the extra lesion however would not have influenced the patient's stage. Example images for one such patient is given in Fig. 2.

Only minimal variations in TBR and SUVmax and SUVmean were observed between the three list-mode acquisitions, with almost perfect agreement (Krippendorf's $\alpha=0.99$ ) between them, suggesting that shorter acquisitions are adequate for routine clinical purposes. We find the shorter acquisitions to be of slightly lower subjective image quality, although such subjective impressions were not the focus of this present study, but rather the aim was to quantify the magnitude of any clinical effect such shorteracquisition protocols may have. While such acquisitions may be less aesthetically pleasing, we find no evidence of clinical detriment. We contend that while a modestly higher background noise was observed in the $30 \mathrm{~s} / \mathrm{bp}$ images (as exemplified by Fig. 3), this was not associated with any objective disadvantage.

Given the known relationship between applied radiopharmaceutical dose, acquisition time and count statistics, by analogy, our data can also be interpreted as supportive of a reduction in applied activity in $\mathrm{dPET} / \mathrm{CT}$. Indeed, with a mean effective dose (excluding the CT component) of $4.9 \mathrm{mSv}$ (Table 1), the $30 \mathrm{~s} / \mathrm{bp}$ reconstructions are equivalent to a $75 \%$ reduction in dose, or $3.7 \mathrm{mSv}$.

We find a small variability in TBR $(0.79 \%)$ when comparing the $30 \mathrm{~s}$ to the $2 \mathrm{~min} / \mathrm{bp}$ reconstructions. Likewise, we find a smaller variability for the parameter SUVmean $(0.35 \%)$ than for SUVmax $(0.77 \%)$. In contrast to previous 
Fig. 2 Example of lesion missed at shorter acquisition (30 s/bp) but easily identified at $1 \mathrm{~min}$ and $2 \mathrm{~min} / \mathrm{bp}$ list-mode acquisition. This retropharyngeal lymph node (blue arrow) was overlooked owing to increased noise in the $30 \mathrm{~s} / \mathrm{bp}$ acquisitions, and is much better delineated at $2 \mathrm{~min}$ and $1 \mathrm{~min} /$ bp. The top row represents the PET data, the bottom row fusion of PET and CT. Bottom right shows colour look-up table (scale 0-8 SUV)
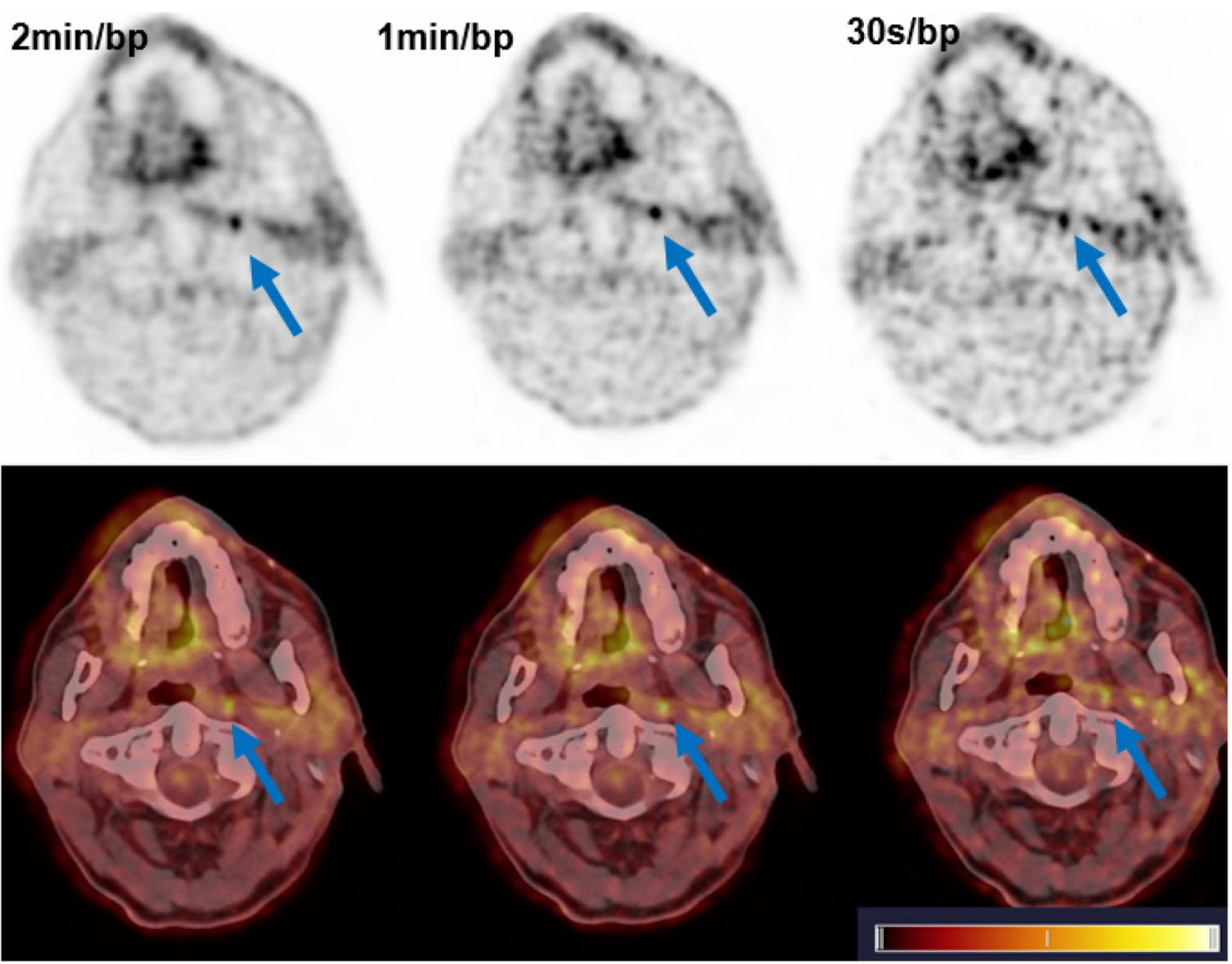

Fig. 3 Example of increased noise for the $30 \mathrm{~s} / \mathrm{bp}$ acquisitions in the maximum intensity projection images (MIP), left most image $2 \mathrm{~min} / \mathrm{bp}$, middle $1 \mathrm{~min} / \mathrm{bp}$, rightmost $30 \mathrm{~s} / \mathrm{bp}$. Note that the increased noise, while subjectively reducing image quality, does not influence the detectability of mediastinal and cervical lymph node lesions in this patient
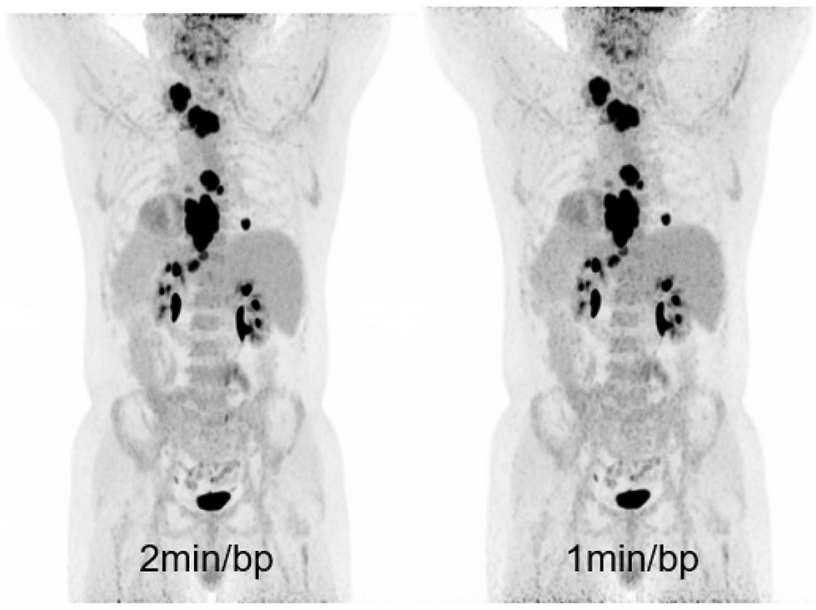

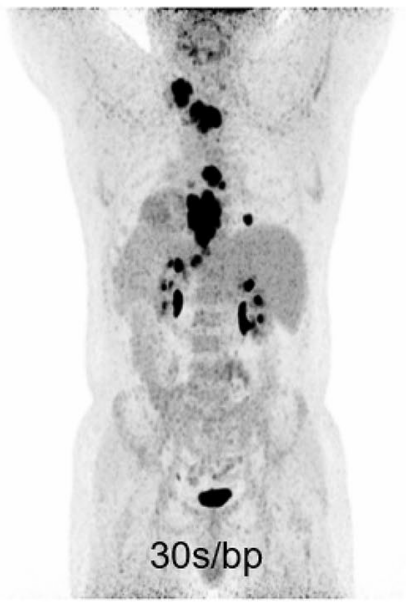

studies comparing the influence of shorter acquisition on SUVmax and SUVmean in a digital system [13] we find no statistically significant differences in SUVmax and TBR between the longer ( $2 \mathrm{~min}$ ) and shorter (30 s) acquisitions. In Fig. 4 we show the SUVmax for the different acquisition durations. Shortening image acquisition durations broadens intensity distributions and should shift the supremum (of a set of values) found within a VOI towards higher values. Furthermore, low counts give rise to tail-heavy distributions in images reconstructed using OSEM and PSF-based algorithms [14], and the choice of reconstruction algorithm can have a significant effect on lesion quantification [13] Therefore, SUVmax, by definition the supremum, is expected to rise when lowering image count $[14,15]$. However, when considering all SUVmax values found in our study, no clear differences can be seen between images for different listmode acquisitions.

As described in the introduction, digital PET/CT represents a step forward in molecular imaging, with increasing numbers of publications confirming the favourable performance characteristics for such scans in comparison to previous-generation analogue systems $[1,16]$, including increased lesion detection and sensitivity $[1,3,4,13,17]$. Previous authors have reported head-to-head studies comparing digital and analogue systems, showing both improved image quality and upstaging of individuals [10], as well as 


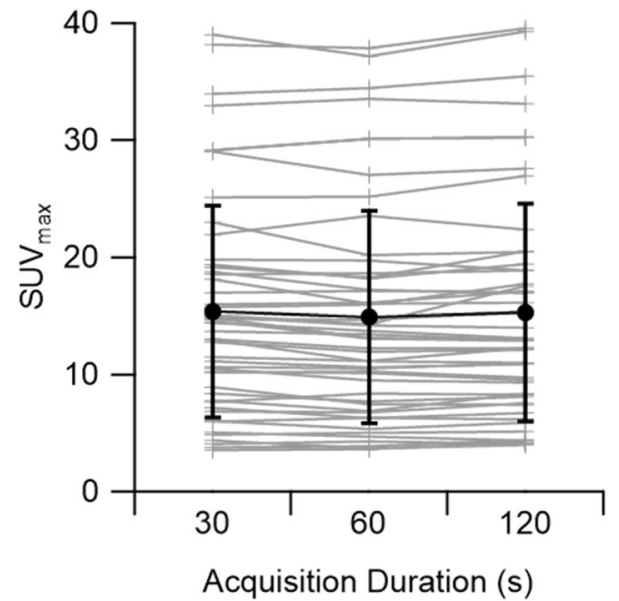

Fig. 4 SUVmax for each lesion by acquisition duration (light grey). In bold (black) is the mean SUVmax \pm standard deviation

improved recovery (as shown by higher SUV values) and lesion sharpness $[3,6]$. Traditionally, shorter list-mode acquisitions have been associated with decreased lesion detection, as most recently shown for $\left[{ }^{68} \mathrm{Ga}\right] \mathrm{Ga}$-PSMA-11 PET/CT by Rauscher et al. (using an analogue scanner) [18] with $1 \mathrm{~min} / \mathrm{bp}$ reconstructions (equivalent to $1 / 3^{\text {rd }}$ of the standard) associated with reduced detection of up to $36 \%$ of lesions. We show here that applied dose reduction or reduction in acquisition time of $75 \%$ is feasible for the combination of $\left[{ }^{18} \mathrm{~F}\right]-\mathrm{FDG}$ and digital PET/CT without detriment with respect to lesion detection. Whereas extant procedural guidelines report protocols and standards for examinations for analogue scanners, the influence of digital PET/CT on list-mode acquisition length and applied dose has not been adequately addressed [7], increasing further the clinical importance of the topic.

Only a small number of publications considering these issues are available in the literature, and only few consider the impact of recent developments in PET technology. For example, Sonni et al. consider the influence of shorter acquisitions with a digital system on image quality (measured on a subjective 5-point scale), but do not report the influence on lesion detection [19]. In the context of combined PET/MRI scanners, multiple publications demonstrate the feasibility of reducing the $\left[{ }^{18} \mathrm{~F}\right]-\mathrm{FDG}$ dose using solid-state (silicon) photomultipliers [20,21]. Van Sluis et al. consider the influence of shorter acquisitions in a digital PET/CT reporting both phantom [1] and clinical data, albeit in a small cohort of patients $(n=30)$ with no estimate of statistical power [13]. Although the authors of this latter study conclude that dose reduction is acceptable, they nevertheless report downstaging for one patient in this small cohort, albeit without any reported influence on therapeutic choice. Any putative benefit deriving from a small reduction in overall radiation exposure in an examination must be balanced against any potential clinical detriment arising from the misstaging of an established cancer-for example the harms caused by the missing of a contralateral lung nodule in a non-small cell lung cancer patient (NSCLC) far outweigh the benefit of a modest reduction in radiation exposure [22]. In contrast we find no detriment in a similarly small cohort, albeit with statistically defined endpoints and adequate statistical power to test our hypotheses. Larger studies must be performed to confirm this for all cancer types and in all patient groups. In their totality, we urge caution when interpreting these data. The equivalency of shorter acquisitions cannot be established with semi-quantiative lesion uptake or qualitative impression of image quality as the sole basis, and further work using clinically defined endpoints are required. The issue is therefore far from being exhaustively investigated, and prospective, randomized studies with larger cohorts are required to determine which patients can safely receive these shorter acquisitions.

Radiological protection concerns, however, are not the sole motivation of applied radioactivity dose reduction. Given the increasing utilisation of PET/CT for monitoring of therapeutics or treatment response, patients are increasingly re-referred for multiple restaging examinations, making accumulated radiation burden of potential relevance, particularly in neonates and infants. Given the limited supply of some radiopharmaceuticals, particulary those with short half-lives [23], we consider that reduction of dose/patient and faster examinations to be of considerable importance in increasing access to nuclear medicine examinations, where average post-referral waiting times for oncological PET/CT can be as high as 14 weeks in some jurisdications [24].

While longer acquisition times traditionally represent a balance between acquisition time and reduction in applied radiation dose, longer acquisitions can be associated with increased motion artefact, and point toward another key advantage in modern scanner design using proprietary endexpiratory PET acquisition for the reduction in respiratory motion artefact [25]. Likewise, while not the subject of this enquiry, the ability to increase numbers of patients examined through shorter-acquisition time is of considerable economic importance in an era of rapidly increasing utilisation of nuclear and molecular medicine imaging techniques.

We note some weaknesses with our study. Firstly, these initial experiences with a new scanner type are in $n=21$ patients, although, in contrast to similar studies, a sample size calculation confirmed the adequacy of this cohort size to answer our hypothesis with adequate statistical power. Nevertheless, these findings must be considered preliminary and interpreted alongside the other similarly small cohorts hitherto published. We use clinically routine and vendor recommended reconstruction methods, EARL-accredited protocols may be better suited to quantitative PET studies in a 
multicentre setting [9]. However, the aim of our study was to show differences in vision detectability, and our chosen acquisition protocol was optimized for this in a clinical setting. We did not assess the influence of lesion size and note that in one case a small lesion was missed (Fig. 2). Previous studies report increased signal recovery from small structures in digital PET/CT systems [26], and further studies are needed to investigate the impact of shorter examinations or low dose protocols on small or low-uptake lesions. Reader bias was reduced using reviewers who had not seen the cases prior to the study. Furthermore, scans were read in randomized and blinded fashion. Few studies confirm the magnitude of any recall bias effect or confirm the optimum time between scan reading [27], although longer waiting periods may be beneficial. Instead, we highlight that scans were read starting with $30 \mathrm{~s} / \mathrm{bp}$ (the images of lowest quality) and in randomized order with a pragmatic $48 \mathrm{~h}$ waiting period in between, limiting the impact of any recall bias inanycase. Other methods of reducing bias, such as using different readers for each list-mode reconstruction are not applicable to this study, where the magnitude of the interobserver agreement is likely larger than the magnitude of the absolute difference between scans (Krippendorf's $\alpha=0.99$ in this study, inter-observer agreement in FDG PET $\kappa=0.69-0.79$ [28]). Finally, institutional ethics board permission afforded anonymous and retrospective data acquisition: further studies, ideally of prospective design, are required to confirm the clinical non-inferiority of images acquired either with shorter-acquisition times, or by analogy lower radiopharmaceutical doses. Previous studies demonstrate that dose reduction is only feasible in non-obese individuals [20]. Our study included only two individuals with BMI $>30 \mathrm{~kg} / \mathrm{m}^{2}$, and none with class II obesity (BMI $>35 \mathrm{~kg} / \mathrm{m}^{2}$ ). Further studies in these population groups are required. Caution must be taken when generalising these results to other radiotracers, and further studies for non-oncological PET/CT (for example in PET for neurodegeneration) and using other tracers are required.

\section{Conclusion}

We demonstrate that shorter acquisitions, or by analogy reduction in applied activity by as much as $75 \%$ is clinically feasible with no clear clinical detriment identified, using a digital PET/CT system. Agreement between these shorter acquisitions and the routine standard-of-care protocol is almost perfect (Krippendorf's $\alpha=0.99$ ) both for lesion detection and tumour-to-background ratio. A small variability in SUVmax, SUVmean and TBR was seen $(<1 \%)$, with no clear relationship between shorter-acquisition times and variability. Our encouraging findings need to be confirmed by larger studies and different clinical settings.

\section{Ethical statement}

The authors report no conflict of interest. A waiver was granted by the regional ethics committee for the retrospective, anonymous analysis of patient data (Req-2020-00678). The study was performed in accordance with the declaration of Helsinki. No funding was obtained.

Funding Open Access funding provided by Universität Bern.

Open Access This article is licensed under a Creative Commons Attribution 4.0 International License, which permits use, sharing, adaptation, distribution and reproduction in any medium or format, as long as you give appropriate credit to the original author(s) and the source, provide a link to the Creative Commons licence, and indicate if changes were made. The images or other third party material in this article are included in the article's Creative Commons licence, unless indicated otherwise in a credit line to the material. If material is not included in the article's Creative Commons licence and your intended use is not permitted by statutory regulation or exceeds the permitted use, you will need to obtain permission directly from the copyright holder. To view a copy of this licence, visit http://creativecommons.org/licenses/by/4.0/.

\section{References}

1. van Sluis JJ, de Jong J, Schaar J, Noordzij W, van Snick P, Dierckx $\mathrm{R}$, et al. Performance characteristics of the digital biograph vision PET/CT system. J Nucl Med. 2019;60(7):1031-6. https://doi. org/10.2967/jnumed.118.215418.

2. Surti S, Viswanath V, Daube-Witherspoom ME, Conti M, Casey ME, Karp JS. Benefit of improved performance with state-of-the art digital PET/CT for lesion detection in oncology. J Nucl Med. 2020. https://doi.org/10.2967/jnumed.120.242305.

3. Nguyen NC, Vercher-Conejero JL, Sattar A, Miller MA, Maniawski PJ, Jordan DW, et al. Image quality and diagnostic performance of a digital PET prototype in patients with oncologic diseases: initial experience and comparison with analog PET. J Nucl Med. 2015;56:1378-85. https://doi.org/10.2967/jnume d.114.148338.

4. Lopez-Mora DA, Flotats A, Fuentes-Ocampo F, Camacho V, Fernandez A, Ruiz A, et al. Comparison of image quality and lesion detection between digital and analog PET/CT. Eur J Nucl Med Mol Imag. 2019;46:1383-90. https://doi.org/10.1007/s0025 9-019-4260-z.

5. Fuentes-Ocampo F, Lopez-Mora DA, Flotats A, Paillahueque G, Camacho V, Duch J, et al. Digital vs analog PET/CT: intrasubject comparison of the SUVmax in target lesions and reference regions. Eur J Nucl Med Mol Imag. 2019;46(8):1745-50. https:// doi.org/10.1007/s00259-018-4256-0.

6. Alberts I, Prenosil G, Sachpekidis C, Weitzel T, Shi K, Rominger A, et al. Digital versus analogue PET in [(68)Ga]Ga-PSMA-11 PET/CT for recurrent prostate cancer: a matched-pair comparison. Eur J Nucl Med Mol Imag. 2020;47(3):614-23. https://doi. org/10.1007/s00259-019-04630-y.

7. Boellaard R, Delgado-Bolton R, Oyen WJG, Giammarile F, Tatsch $\mathrm{K}$, Eschner W, et al. FDG PET/CT: EANM procedure guidelines for tumour imaging: version 2.0. Eur J Nucl Med Mol Imag. 2015;42:328-54. https://doi.org/10.1007/s00259-014-2961-x. 
8. van Sluis J, Boellaard R, Dierckx R, Stormezand GN, Glaudemans A, Noordzij W. Image quality and activity optimization in oncologic (18)F-FDG PET using the digital biograph vision PET/ CT system. J Nucl Med. 2020;61:764-71. https://doi.org/10.2967/ jnumed.119.234351.

9. Koopman D, Jager PL, Slump CH, Knollema S, van Dalen JA. SUV variability in EARL-accredited conventional and digital PET. EJNMMI Res. 2019;9:106. https://doi.org/10.1186/s1355 0-019-0569-7.

10. Koopman D, van Dalen JA, Stevens H, Slump CH, Knollema S, Jager PL. Performance of digital PET compared to high-resolution conventional PET in patients with cancer. J Nucl Med. 2020;61(10):1448-54. https://doi.org/10.2967/jnumed.119.23810 5 .

11. Lee H, Paeng JC, Hong SH, Yoo HJ, Cheon GJ, Lee DS, et al. Appropriate margin thresholds for isocontour metabolic volumetry of fluorine-18 fluorodeoxyglucose PET in sarcoma: a hybrid PET/MRI study. Nucl Med Commun. 2016;37:1088-94. https:// doi.org/10.1097/MNM.0000000000000561.

12. Wahl RL, Jacene H, Kasamon Y, Lodge MA. From RECIST to PERCIST: evolving considerations for PET response criteria in solid tumors. J Nucl Med. 2009;50(Suppl 1):122S-S150. https:// doi.org/10.2967/jnumed.108.057307.

13. van Sluis J, Boellaard R, Dierckx RA, Stormezand G, Glaudemans A, Noordzij W. Image quality and activity optimization in oncological (18)F-FDG PET using the digital Biograph Vision PET/ CT. J Nucl Med. 2019. https://doi.org/10.2967/jnumed.119.23435 1 .

14. Prenosil GA, Weitzel T, Furstner M, Hentschel M, Krause T, Cumming $\mathrm{P}$, et al. Towards guidelines to harmonize textural features in PET: Haralick textural features vary with image noise, but exposure-invariant domains enable comparable PET radiomics. PLoS ONE. 2020;15:e0229560. https://doi.org/10.1371/journ al.pone. 0229560 .

15. Lodge MA, Chaudhry MA, Wahl RL. Noise considerations for PET quantification using maximum and peak standardized uptake value. J Nucl Med. 2012;53:1041-7. https://doi.org/10.2967/ jnumed.111.101733.

16. Rausch I, Ruiz A, Valverde-Pascual I, Cal-Gonzalez J, Beyer T, Carrio I. Performance evaluation of the vereos PET/CT system according to the NEMA NU2-2012 standard. J Nucl Med. 2019;60:561-7. https://doi.org/10.2967/jnumed.118.215541.

17. Schillaci O, Urbano N. Digital PET/CT: a new intriguing chance for clinical nuclear medicine and personalized molecular imaging. Eur J Nucl Med Mol Imag. 2019. https://doi.org/10.1007/s0025 9-019-04300-z.

18. Rauscher I, Fendler WP, Hope TA, Quon A, Nekolla SG, Calais J, et al. Can the injected dose be reduced in (68)Ga-PSMA-11 PET/ $\mathrm{CT}$ while maintaining high image quality for lesion detection?
J Nucl Med. 2020;61:189-93. https://doi.org/10.2967/jnume d.119.227207.

19. Sonni I, Baratto L, Park S, Hatami N, Srinivas S, Davidzon G, et al. Initial experience with a SiPM-based PET/CT scanner: influence of acquisition time on image quality. EJNMMI Phys. 2018;5:9. https://doi.org/10.1186/s40658-018-0207-x.

20. Sekine T, Delso G, Zeimpekis KG, de Galiza BF, ter Voert EEGW, Huellner M, et al. Reduction of 18F-FDG dose in clinical PET/ MR imaging by using silicon photomultiplier detectors. Radiology. 2017;286:249-59. https://doi.org/10.1148/radiol.2017162305

21. Sah BR, Ghafoor S, Burger IA, Ter Voert E, Sekine T, Delso G, et al. Feasibility of (18)F-FDG dose reductions in breast cancer PET/MRI. J Nucl Med. 2018;59:1817-22. https://doi.org/10.2967/ jnumed.118.209007.

22. McCready VR, Dizdarevic S, Beyer T. Lesion detection and administered activity. J Nucl Med. 2020. https://doi.org/10.2967/ jnumed.120.244020.

23. Mueller D, Fuchs A, Leshch Y, Proehl M. The shortage of approved $68 \mathrm{Ge} / 68 \mathrm{Ga}$ generators-incoming material inspection and GMP compliant use of non-approved generators. J Nucl Med. 2019;60:1059.

24. Beyer T, Czernin J, Freudenberg LS. Variations in clinical PET/ CT operations: results of an international survey of active PET/ CT users. J Nucl Med. 2011;52:303-10.

25. Hamill J, Meier JG, Betancourt Cuellar SL, Sabloff B, Erasmus JJ, Mawlawi O. Improved alignment of PET and CT images in whole-body PET/CT in cases of respiratory motion during CT. J Nucl Med. 2020. https://doi.org/10.2967/jnumed.119.235804.

26. Meyer M, Allenbach G, Nicod Lalonde M, Schaefer N, Prior JO, Gnesin S. Increased (18)F-FDG signal recovery from small physiological structures in digital PET/CT and application to the pituitary gland. Sci Rep. 2020;10:368. https://doi.org/10.1038/ s41598-019-57313-x.

27. Karla KE, Edith MM, Myrna CBG, Diana P, Tara S, Sonia Betancourt $\mathrm{C}$, et al. Radiologists remember mountains better than radiographs, or do they? J Med Imag. 2015;3:1-9. https://doi. org/10.1117/1.JMI.3.1.011005.

28. Marcus C, Ciarallo A, Tahari AK, Mena E, Koch W, Wahl RL, et al. Head and neck PET/CT: therapy response interpretation criteria (Hopkins Criteria)-interreader reliability, accuracy, and survival outcomes. J Nucl Med. 2014;55:1411-6. https://doi. org/10.2967/jnumed.113.136796.

Publisher's Note Springer Nature remains neutral with regard to jurisdictional claims in published maps and institutional affiliations. 